Message from the Editor

\title{
Rating an academic journal-indexing and impact factor
}

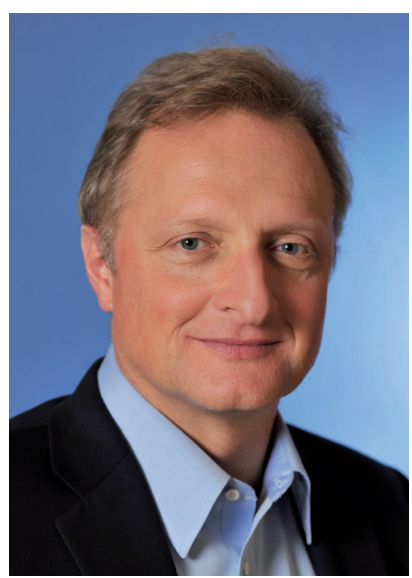

Jens Chapman
Upon the conclusion of its first year of existence many colleagues have asked how EBSJ is faring on the road toward becoming a recognized academic entity. To this end there are two major recognized elements: Indexing and Impact Factor. On the part of the first entity EBSJ can proclaim a first major victory. EBSJ has been accepted for indexing in PubMed $^{\circledR}$, the largest search engine for medical literature in the world. This step will include retroactive recognition of our back issues. Please join me in thanking our publishers, staff, and all participating authors, reviewers, and editors who have made this possible. Our quest for inclusion in other search engines will continue with renewed energy. The second commonly recurring question is what is the impact factor of a new journal, how is it calculated, and when EBSJ would be rated in this fashion? Since these questions are asked often, I thought it might be interesting to review this subject briefly in the editor's message.

The impact factor (IF) is a widely accepted calculation used to measure the relative importance or impact of science journals. It is based on the average number of times published articles are cited up to 2 years after publication. The IF is calculated by dividing the number of current year citations to the source items published in that journal during the previous 2 years.

For example, the 2010 IF for a journal is calculated as follows:

$$
\mathbf{2 0 1 0} \mathbf{I F}=\frac{\mathbf{A}\left(\sum\right. \text { articles published in 2008-2009 cited in indexed journals during 2010) }}{\mathbf{B} \text { (number of 'citable items' published in 2008-2009) }}
$$

A journal with a high-IF ratio is perceived to have significant impact in its field.

However, there are some considerations one must take into account when looking at a calculated IF for a journal.

- First, the journal has to be indexed in order for the IF to be calculated.

- Second, journals with larger readership exposure fare better.

- Third, the more narrow the subject field the better.

- Fourth, formal review or reference articles do better.

- Finally, organizations with self-referencing option also do better.

When taking these considerations into account, we find that the IF calculation is more of a marketing tool rather than a direct reflection of the journals quality.

\section{So then what might be the 'true' impact of a journal and the evidence it contains?}

Let's consider how research quality, and how it is reported, impacts the bigger picture of health care and reimbursement. 
Globally, a wide variety of stakeholders are progressively evaluating and critically appraising medical literature. Increasingly, published evidence in our field is being examined to create policy and reimbursement decisions across the world. In our field of interest stakeholders include our patients, the international community of spine surgeons, thirdparty payers, the AO at large, allied personnel involved in care of patients with spine disease, other academic organizations, governmental agencies, and others with interests in evidence-based practice and technology assessment.

EBSJ's mission revolves around assisting the global spine community to discover, develop, and disseminate research findings in ways that no other journal in the market is currently doing. The goal is to significantly enhance the quality of research and evidence that is available for evaluation. It has never been more important to raise the standards for the quality of evidence than now. In the end, it is the quality, not the IF that will exert the most influence on our professional credibility and how we are reimbursed.

\section{Improving the evidence will not happen overnight}

This is where EBSJ plays an important role in assisting with creating true impact on medical care and policymaking. EBSJ encourages authors and researchers across the globe to learn about research quality and reporting by submitting studies that may not be perfect. Why you may ask? Because these are perfect learning opportunities for the authors and readers alike.

All the original research submitted to EBSJ undergoes a PhD-level methods review. Authors are given formative feedback for study improvement and reporting. The level of evidence (LoE) for each article and Editorial Staff perspective help facilitate understanding and application to future research.

While the "impact factor" may get your name in lights, the quality of information and evidence is what may have the more extensive true impact when it comes to how we care for our patients and the credibility of the research in our field-and increasingly how we, as spine-care professionals, are paid for the work that we do.

The mission of AOSpine revolves around the creation and dissemination of knowledge about best practices for providing care. AOSpine serves the growing global community of spine-care professionals by offering world-class education and services in order to advance the effectiveness of care. EBSJ is the perfect venue for assisting with the AOSpine mission and raising the standards.

With that said, we are proud to present our first issue of 2011.

Yours,

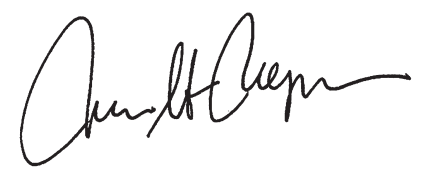

Jens Chapman, Editor-in-Chief

The Editorial Board of EBSJ takes the opportunity to thank Dr Marinus DeKleuver for his past contributions to EBSJ as the AOSpine Europe regional editor. His efforts this past year have been greatly appreciated. We also extend a warm welcome to Dr Guiseppe Barbagallo to EBSJ as the incoming regional editor for AOSpine Europe. We are looking forward to Dr Barbagallo's contributions to the upcoming issues of EBSJ. 